\title{
Intravascular Ultrasound Evaluation of Interwoven Nitinol Stents at Implant
}

Frank J Arena* and Frank A Arena

Northoaks Medical Center, Hammond, Lousiana, USA

\begin{abstract}
Purpose: To evaluate the performance of Interwoven Nitinol Stents placed in the Superficial Femoral and Popliteal arteries using Intravascular Ultrasound (IVUS).

Methods: 37 stented segments in 34 patients whose procedures involved using IVUS during their periphera angioplasties were retrospectively examined. Twenty seven $5 \mathrm{~mm}$ stented segments and ten $6 \mathrm{~mm}$ segments were measured. The IVUS study showing each stented segment was measured at ten approximately equal distances. At each of these points the size of the lumen was measured. The area inside the stents, as well as the minimum and maximum diameters across the stent were determined. The minimal area from each segment was also obtained. These areas were averaged and the stent expansion ratio (SER), average in stent area/maximal stent area was determined for each segment. The minimum and maximum diameters in each segment were also determined and the Radial Stent Symmetry Index (RSSI), minimum/maximum stent diameter was calculated for each segment.
\end{abstract}

Results: The $5 \mathrm{~mm}$ Interwoven nitinol stent group achieved an SER of $96.5 \%$. The $6 \mathrm{~mm}$ group achieved an SER of $87.83 \%$. The minimal SER measurement among the $5 \mathrm{~mm}$ stents averaged $76.67 \%$ and the $6 \mathrm{~mm}$ stents $65.71 \%$. The RSSI in the $5 \mathrm{~mm}$ segments were $90.49 \%$, while the $6 \mathrm{~mm}$ group was $88.80 \%$.

Conclusions: Interwoven Nitinol stents perform well at implant with consistent deployment areas across multiple stent sizes when used in the SFA and Popliteal arteries. RSSI calculations were generally consistent with a relatively round stent deployment.

Keywords: Intravascular ultrasound; Stent; Peripheral; Angioplasty

\section{Introduction}

Stenting of the Superficial Femoral and Popliteal arteries have become more commonplace over the last 2 decades. Performance of these stents have generally been determined by clinical patency and restenosis by external Doppler ultrasound and repeat angiography [1]. Very little information exists regarding how these peripheral stents appear and perform immediately after deployment. Intravascular ultrasound (IVUS) has become a useful tool in the coronary anatomy to evaluate stents for adequate deployment and to evaluate the artery for hidden issues that may affect patency such as dissection [2,3]. Adequacy of deployment and stent size at the time of implant has been associated with better long term outcome in the coronary anatomy [4-6]. The use of IVUS technology in the peripheral circulation is becoming more commonplace [7]. This data provides significant anatomic information felt to be useful during complex peripheral interventions, although clinical trial data is lacking. During peripheral artery procedures in the SFA and Popliteal, (especially when working in calcified arteries) anecdotally, it is not uncommon to see traditional tubular nitinol stents compress from external forces and fail to maintain their shapes at implant [3] (Figure 1). Few studies have been performed to assess the impact of this phenomenon $[3,8]$.

In recent years a new stent design, interwoven nitinol has been available for use in the SFA and Popliteal arteries [9]. This design has several theoretical advantages including substantially increased radial strength as well as better compliance to the forces of longitudinal compression and extension when compared to traditional tubular nitinol stents [10]. As of the writing of this paper no information exists in the literature documenting the deployment performance of this stent design at implant. We retrospectively evaluated the intravascular ultrasound results on 37 consecutive stented segments in patients

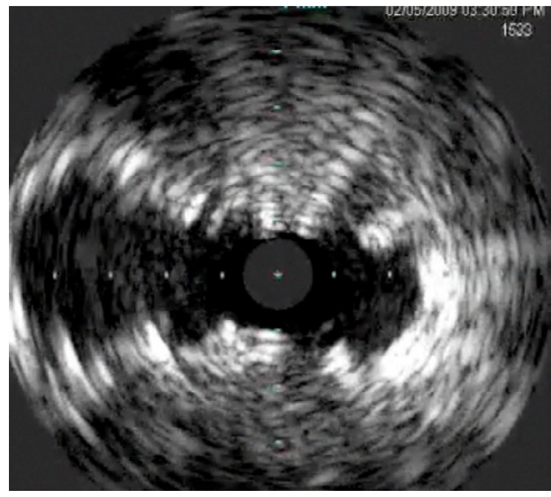

Figure 1: An 8mm Traditional nitinol stent successfully post dilated with $6 \mathrm{~mm}$ balloon to $15 \mathrm{~atm}$. showing example of external compression.

who had IVUS of interwoven nitinol stents placed in their SFA and/or Popliteal arteries in our lab. The stents examined were all between 5 and $6 \mathrm{~mm}$ in size. These stents were placed as part of the routine peripheral

*Corresponding author: Frank J. Arena, 54 Preserve Lane, Mandeville, Louisiana 70471, IVUS Interwoven Nitinol at implant, USA, E-mail: farena4@att.net

Received August 22, 2013; Accepted September 09, 2013; Published September 11,2013

Citation: Arena FJ, Arena FA (2013) Intravascular Ultrasound Evaluation of Interwoven Nitinol Stents at Implant. J Vasc Med Surg 1: 116 doi: 10.4172/23296925.1000116

Copyright: ( 2013 Arena FJ, et al. This is an open-access article distributed under the terms of the Creative Commons Attribution License, which permits unrestricted use, distribution, and reproduction in any medium, provided the original author and source are credited. 
intervention. There were 3 TASC B, 20 TASC C and 11 TASC D patients who made up the study group. The average Rutherford class was 4.1 for the combined group (Table 1 and 2).

\section{Methods}

In all 37 stented segments in 34 patients using the Supera Stent, IDEV Technologies (Webster, Tx. USA), were evaluated using the Volcano Eagle Eye Gold, (San Diego, Ca. USA) IVUS system during the normal course of their peripheral angioplasty procedures. The Volcano Eagle Eye Gold is a .014 wire based system used with manual pullback during the IVUS examination. The Ivus examinations in this study were all intra-procedure and post procedure examinations and were performed over concerns about adequacy of deployment and dissection as well as disease distal to the stented segment. Stent sizes were chosen based on angiography and clinical judgement. There were twenty seven,

\begin{tabular}{|c|c|c|c|}
\hline $\mathrm{Pt}$ & TASC & RUTHERFORD & STENT LENGTH \\
\hline 1 & $\mathrm{D}$ & 3 & $5 \mathrm{~mm} \times 30 \mathrm{CM}$ \\
\hline 2 & $\mathrm{D}$ & 4 & $5 \mathrm{~mm} \times 22 \mathrm{CM}$ \\
\hline 3 & $\mathrm{D}$ & 4 & $5 \mathrm{~mm} \times 16 \mathrm{CM}$ \\
\hline 4 & B & 3 & $5 \mathrm{~mm} \times 12 \mathrm{CM}$ \\
\hline 5 & C & 4 & $5 \mathrm{~mm} \times 23 \mathrm{CM}$ \\
\hline 6 & C & 4 & $5 \mathrm{~mm} \times 24 \mathrm{CM}$ \\
\hline 7 & $\mathrm{D}$ & 5 & $5 \mathrm{~mm} \times 12 \mathrm{CM}$ \\
\hline 8 & $B$ & 6 & $5 \mathrm{~mm} \times 10 \mathrm{CM}$ \\
\hline 9 & $\mathrm{D}$ & 5 & $5 \mathrm{~mm} \times 36 \mathrm{CM}$ \\
\hline 10 & $\mathrm{C}$ & 4 & $5 \mathrm{~mm} \times 18 \mathrm{CM}$ \\
\hline 11 & $\mathrm{C}$ & 4 & $5 \mathrm{~mm} \times 22 \mathrm{CM}$ \\
\hline 12 & $D$ & 4 & $5 \mathrm{~mm} \times 34 \mathrm{CM}$ \\
\hline 13 & $\mathrm{C}$ & 4 & $5 \mathrm{~mm} \times 18 \mathrm{CM}$ \\
\hline 14 & $\mathrm{C}$ & 4 & $5 \mathrm{~mm} \times 12 \mathrm{CM}$ \\
\hline 15 & $\mathrm{D}$ & 5 & $5 \mathrm{~mm} \times 18 \mathrm{CM}$ \\
\hline 16 & $\mathrm{D}$ & 4 & $5 \mathrm{~mm} \times 30 \mathrm{CM}$ \\
\hline 17 & C & 5 & $5 \mathrm{~mm} \times 30 \mathrm{CM}$ \\
\hline 18 & $\mathrm{C}$ & 4 & $5 \mathrm{~mm} \times 24 \mathrm{CM}$ \\
\hline 19 & $\mathrm{C}$ & 4 & $5 \mathrm{~mm} \times 38 \mathrm{CM}$ \\
\hline 20 & $\mathrm{C}$ & 5 & $5 \mathrm{~mm} \times 21 \mathrm{CM}$ \\
\hline 21 & $\mathrm{C}$ & 5 & $5 \mathrm{~mm} \times 24 \mathrm{CM}$ \\
\hline 22 & $\mathrm{C}$ & 3 & $5 \mathrm{~mm} \times 16 \mathrm{CM}$ \\
\hline 23 & C & 3 & $5 \mathrm{~mm} \times 24 \mathrm{CM}$ \\
\hline 24 & $D$ & 5 & $5 \mathrm{~mm} \times 46 \mathrm{CM}$ \\
\hline 25 & $D$ & 4 & $5 \mathrm{~mm} \times 24 \mathrm{CM}$ \\
\hline 26 & C & 4 & $5 \mathrm{~mm} \times 24 \mathrm{CM}$ \\
\hline 27 & B & 3 & $5 \mathrm{~mm} \times 12 \mathrm{CM}$ \\
\hline
\end{tabular}

Table 1: Patient procedure and stent characteristics $5 \mathrm{~mm}$ group.

\begin{tabular}{|c|c|c|c|}
\hline Pt & TASC & RUTHERFORD & STENT LENGTH \\
\hline 1 & C & 4 & $6 \mathrm{~mm} \times 30 \mathrm{CM}$ \\
\hline 2 & $\mathrm{C}$ & 6 & $6 \mathrm{~mm} \times 10 \mathrm{CM}$ \\
\hline 3 & $\mathrm{C}$ & 3 & $6 \mathrm{~mm} \times 20 \mathrm{CM}$ \\
\hline 4 & $\mathrm{D}$ & 4 & $6 \mathrm{~mm} \times 22 \mathrm{CM}$ \\
\hline 5 & $\mathrm{C}$ & 4 & $6 \mathrm{~mm} \times 8 \mathrm{CM}$ \\
\hline 6 & $\mathrm{D}$ & 4 & $6 \mathrm{~mm} \times 8 \mathrm{CM}$ \\
\hline 7 & $\mathrm{C}$ & 4 & $6 \mathrm{~mm} \times 12 \mathrm{CM}$ \\
\hline 8 & $\mathrm{C}$ & 4 & $6 \mathrm{~mm} \times 28 \mathrm{CM}$ \\
\hline 9 & $\mathrm{C}$ & 4 & $6 \mathrm{~mm} \times 30 \mathrm{CM}$ \\
\hline 10 & $\mathrm{C}$ & 4 & $6 \mathrm{~mm} \times 24 \mathrm{CM}$ \\
\hline
\end{tabular}

Table 2: Patient procedure and stent characteristics $6 \mathrm{~mm}$ group.
$5 \mathrm{~mm}$ stents and ten, $6 \mathrm{~mm}$ stented segments measured. Three patients had limbs stented with both 5 and $6 \mathrm{~mm}$ stents, these were treated as separate examinations. The cases were collected and retrospectively examined. We split the IVUS evaluation of each segment into ten approximately equal distances using the frame number included in the video recording. For example, if 1000 frames were in the recording we would measure at 100,200 etc. Since this was a retrospective evaluation of routine cases, measurements at exact distances were not possible. At each of the 10 positions in the recording the size of the lumen was measured using a Volcano System 5 workstation. The area inside the stents as well as the minimum and maximum diameters across the stents were determined. The entire IVUS recording was reviewed to find the minimum luminal area and that measurement was also recorded. The area measurement results in each segment were combined and the Stent Expansion Ratio (SER) for each group (5 and $6 \mathrm{~mm}$ stents) was calculated. The minimum area in each segment was averaged and the minimum SER was also determined for each group (Table 3 and 4). In addition the minimum and maximum diameter measurements were combined and the Radial Stent Symmetry Index (RSSI) for each group was determined as an estimate for the roundness of the deployed stents (Table 5 and 6).

\section{Results}

The average SER in the $5 \mathrm{~mm}$ interwoven nitinol stent group was $96.5 \%$. In the $6 \mathrm{~mm}$ stent group the calculation was $87.83 \%$. The average minimal SER of each segment in the $5 \mathrm{~mm}$ stents was $76.67 \%$. The average minimum for the group of $6 \mathrm{~mm}$ stents were $65.71 \%$. The RSSI in the $5 \mathrm{~mm}$ group was $90.49 \%$, The calculation for the $6 \mathrm{~mm}$ group was $88.80 \%$.

\section{Discussion}

Anecdotally, intravascular ultrasound has shown instances of significant external compression despite adequate initial expansion of traditional nitinol stents placed in the SFA and Popliteal arteries (Figure 1). This study supports the clinical observations that interwoven nitinol stents deploy consistently near their theoretical maximum with good resistance to external compression (Figure 2). The relative lack of deployment seen in the $6 \mathrm{~mm}$ cohort may be partially due to external forces but also is likely to be associated with the inability to dilate the vessel to an adequate size to allow full deployment. In our lab the tendency is to go to 18-20 atmospheres with the same size balloon as the stent in the predeployment inflation and using the same balloon

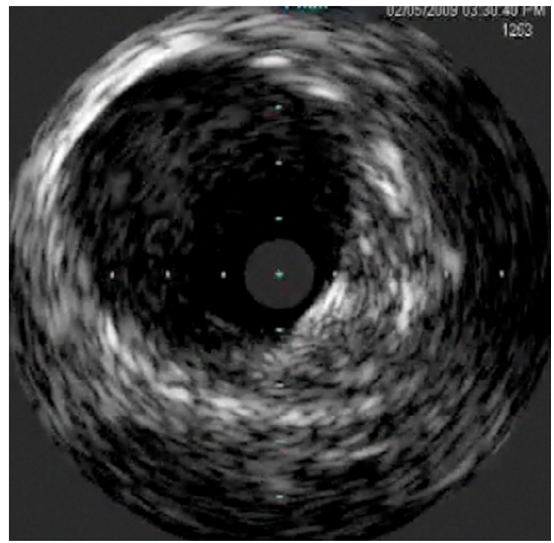

Figure 2: Same artery as figure 1, Interwoven Nitinol stent just distal to stent in figure 1. 
Citation: Arena FJ and Arena FA (2013) Intravascular Ultrasound Evaluation of Interwoven Nitinol Stents at Implant. J Vasc Med Surg 1: 116 doi: 10.4172/2329-6925.1000116

Page 3 of 6

\begin{tabular}{|c|c|c|c|c|c|c|c|c|c|c|c|c|}
\hline PT & 1 & 2 & 3 & 4 & 5 & 6 & 7 & 8 & 9 & 10 & AVE & MIN \\
\hline 1 & 18.3 & 15.6 & 18.8 & 18.7 & 19 & 18.7 & 17.3 & 19.8 & 19.6 & 18.9 & 18.47 & 15.6 \\
\hline 2 & 17.7 & 18.6 & 15.4 & 15 & 18.9 & 21.3 & 19 & 20.1 & 21.8 & 18 & 18.58 & 13.2 \\
\hline 3 & 19 & 17.5 & 21 & 21.3 & 21 & 21.8 & 16.7 & 16.8 & 15.5 & 18.4 & 18.9 & 13.2 \\
\hline 4 & 14.6 & 18 & 17.3 & 20.4 & 20.3 & 18.4 & 20.5 & 20.4 & 22.4 & 19.2 & 19.15 & 14.6 \\
\hline 5 & 17.8 & 18.7 & 15.8 & 14.8 & 14.6 & 14.5 & 18.3 & 16.5 & 17.2 & 17.5 & 16.57 & 12.9 \\
\hline 6 & 22 & 18.9 & 17.8 & 18.4 & 19.7 & 19.5 & 18.9 & 16.3 & 19.7 & 16.6 & 18.78 & 15.2 \\
\hline 7 & 16 & 15.6 & 18.2 & 18.6 & 18.1 & 19.5 & 18.8 & 19.7 & 19.9 & 19.3 & 18.37 & 14 \\
\hline 8 & 18 & 18.2 & 14.4 & 18.3 & 18.4 & 19.3 & 16.8 & 17 & 17.2 & 19.5 & 17.71 & 13.7 \\
\hline 9 & 18.4 & 19.1 & 16.3 & 17.8 & 17.2 & 17.7 & 17.4 & 20.6 & 21.3 & 21.6 & 18.74 & 15.6 \\
\hline 10 & 16.8 & 17.1 & 13.2 & 19.5 & 20 & 17.9 & 21.3 & 19.9 & 19.6 & 21.3 & 18.66 & 13.2 \\
\hline 11 & 17.8 & 17.4 & 14.7 & 18.8 & 17.3 & 18.8 & 18.2 & 17.8 & 19.3 & 18.3 & 17.84 & 14.2 \\
\hline 12 & 20.5 & 20.6 & 19.9 & 18.1 & 19.3 & 18.6 & 16.4 & 19.4 & 20.2 & 19.8 & 19.28 & 15.1 \\
\hline 13 & 19.6 & 18.9 & 19.9 & 22.6 & 19.6 & 22.2 & 23.3 & 20.3 & 19.9 & 17.9 & 20.42 & 17.7 \\
\hline 14 & 23 & 22.7 & 20.2 & 21.2 & 19.6 & 22.1 & 19.5 & 21 & 20.5 & 21.2 & 21.1 & 19.5 \\
\hline 15 & 12.7 & 14.1 & 15.1 & 15.5 & 14.4 & 18 & 19.8 & 19.6 & 20.8 & 18.7 & 16.87 & 12.2 \\
\hline 16 & 20.6 & 19.7 & 18.7 & 18.9 & 20.2 & 20.8 & 19.5 & 20.7 & 21.7 & 18.4 & 19.92 & 18.1 \\
\hline 17 & 15.9 & 22.5 & 21.7 & 18.8 & 21.6 & 20.7 & 20.5 & 22.5 & 19.1 & 23.3 & 20.66 & 15.9 \\
\hline 18 & 18.8 & 18.8 & 15.9 & 16.1 & 18.4 & 17.6 & 16.2 & 17.5 & 18.6 & 20.3 & 17.82 & 13.4 \\
\hline 19 & 19.9 & 21.3 & 21.9 & 23.9 & 23.1 & 20.9 & 17.3 & 19.4 & 21.4 & 20.7 & 20.98 & 17.3 \\
\hline 20 & 21.2 & 16.9 & 19.1 & 17.1 & 18.4 & 17.3 & 22 & 17.4 & 21.4 & 20.8 & 19.16 & 12.7 \\
\hline 21 & 19.3 & 17.2 & 14.3 & 15.2 & 17.5 & 18.5 & 17.1 & 20.5 & 20.2 & 21.3 & 18.11 & 13.8 \\
\hline 22 & 15.7 & 17.8 & 15.1 & 18.1 & 18.2 & 20 & 19.3 & 17.9 & 18.8 & 19.4 & 18.03 & 15.1 \\
\hline 23 & 16.5 & 18.8 & 16.6 & 19.9 & 16.8 & 19.4 & 18.6 & 19.3 & 18.2 & 17.3 & 18.14 & 16.6 \\
\hline 24 & 21.5 & 17.3 & 18.4 & 19.3 & 16.7 & 21.5 & 19.6 & 21.1 & 20.3 & 21.3 & 19.7 & 16.7 \\
\hline 25 & 20.4 & 21.1 & 20.5 & 21.4 & 17 & 19.3 & 16.4 & 19 & 20 & 21.3 & 19.64 & 15.1 \\
\hline 26 & 19.6 & 18.1 & 18.4 & 14.9 & 23.8 & 22.7 & 22.2 & 23.2 & 18.5 & 18.8 & 20.02 & 14.3 \\
\hline 27 & 20.6 & 19.1 & 18.6 & 17.4 & 18.4 & 17.9 & 21.7 & 21.7 & 20.3 & 24.8 & 20.05 & 17.4 \\
\hline
\end{tabular}

Average intraluminal area of $5 \mathrm{~mm}$ Group- $18.95 \mathrm{~mm}^{2}$

Average Minimal area of $5 \mathrm{~mm}$ group $-15.05 \mathrm{~mm}^{2}$

Stent Expansion Ratio- 96.52\%

Minimum Stent Expansion Ratio-76.67\%

Table 3: $5 \mathrm{~mm}$ stent group IVUS area data.

\begin{tabular}{|c|c|c|c|c|c|c|c|c|c|c|c|c|}
\hline PT & 1 & 2 & 3 & 4 & 5 & 6 & 7 & 8 & 9 & 10 & AVE & Min \\
\hline 1 & 24.5 & 25.9 & 16.3 & 27.1 & 25.7 & 27.2 & 32.6 & 27.2 & 22.9 & 25.3 & 25.47 & 15.9 \\
\hline 2 & 21.6 & 24.1 & 22 & 23.6 & 21.4 & 21.9 & 20.4 & 20.4 & 30.1 & 28.3 & 23.38 & 15.4 \\
\hline 3 & 24.8 & 21.7 & 23.3 & 23.2 & 19.4 & 21.8 & 21.7 & 22.6 & 24 & 25 & 22.75 & 19.4 \\
\hline 4 & 28.7 & 23 & 28 & 26.3 & 25.5 & 25.3 & 25.7 & 26.2 & 27.4 & 23.4 & 25.95 & 20.2 \\
\hline 5 & 27.7 & 27.3 & 24.1 & 24.5 & 23 & 24.3 & 26.5 & 25.2 & 25.6 & 25.6 & 25.38 & 22.3 \\
\hline 6 & 20.8 & 22.9 & 20.2 & 23.3 & 24.2 & 22.2 & 29 & 29.3 & 26.9 & 26.6 & 24.54 & 20.2 \\
\hline 7 & 28 & 28.6 & 27.5 & 26.6 & 22.6 & 27.2 & 29.1 & 27.8 & 28.8 & 25.3 & 27.15 & 22 \\
\hline 8 & 19.4 & 21 & 31 & 24.8 & 20.5 & 23.4 & 25.7 & 31.9 & 32.2 & 29.1 & 25.9 & 20.5 \\
\hline 9 & 27.7 & 26.4 & 33.3 & 20.1 & 30 & 32.2 & 25.2 & 17.4 & 20.1 & 23.7 & 25.61 & 13.6 \\
\hline 10 & 23 & 22.8 & 17.6 & 18.1 & 22 & 21.9 & 21.4 & 23.2 & 29.1 & 23 & 22.21 & 16.3 \\
\hline
\end{tabular}

Average luminal area of $6 \mathrm{~mm}$ group- $24.83 \mathrm{~mm}^{2}$

Average minimal luminal area of group-18.58 $\mathrm{mm}^{2}$

Stent Expansion Ratio-87.83\%

Minimal Stent Expansion Ratio-65.71\%

Table 4: $6 \mathrm{~mm}$ stent group IVUS area data.

\begin{tabular}{|c|c|c|c|c|c|c|c|c|c|c|c|c|}
\hline PT & 1 & 2 & 3 & 4 & 5 & 6 & 7 & 8 & 9 & 10 & AVE & RSSI \\
\hline Min & 4.7 & 4.7 & 4.7 & 4.5 & 4.7 & 4.4 & 5 & 5 & 4.8 & 5.4 & 4.79 & \multirow{2}{*}{0.9123} \\
\hline Max & 5.4 & 5 & 5 & 4.9 & 5.1 & 5 & 5.4 & 5.5 & 5.4 & 5.8 & 5.25 & \\
\hline \multirow[t]{2}{*}{2} & 4.6 & 4.3 & 4.6 & 4.7 & 4.7 & 4.7 & 4.4 & 4.9 & 4.8 & 4.8 & 4.65 & \multirow{2}{*}{0.9207} \\
\hline & 5 & 4.6 & 5.1 & 5 & 5.1 & 5 & 5.1 & 5.3 & 5.2 & 5.1 & 5.05 & \\
\hline \multirow[t]{2}{*}{3} & 4.3 & 4.2 & 3.9 & 4 & 4.3 & 4.5 & 4.4 & 4.1 & 4.1 & 4 & 4.18 & \multirow{2}{*}{0.8893} \\
\hline & 4.9 & 5.1 & 4.5 & 4.4 & 4.9 & 5 & 4.8 & 4.6 & 4.4 & 4.4 & 4.7 & \\
\hline
\end{tabular}


Citation: Arena FJ and Arena FA (2013) Intravascular Ultrasound Evaluation of Interwoven Nitinol Stents at Implant. J Vasc Med Surg 1: 116 doi: 10.4172/2329-6925.1000116

Page 4 of 6

\begin{tabular}{|c|c|c|c|c|c|c|c|c|c|c|c|c|}
\hline 4 & 4.3 & 4.7 & 4.2 & 4.1 & 4.7 & 5 & 4.5 & 4.7 & 5 & 4.4 & 4.56 & \multirow{2}{*}{0.8923} \\
\hline & 5 & 5.1 & 4.7 & 4.5 & 5.1 & 5.4 & 5.1 & 5.5 & 5.6 & 5.1 & 5.11 & \\
\hline \multirow[t]{2}{*}{5} & 4.1 & 4.5 & 4.4 & 4.9 & 4.9 & 4.5 & 4.8 & 4.9 & 5.1 & 4.7 & 4.68 & \multirow{2}{*}{0.9052} \\
\hline & 4.5 & 5.2 & 4.9 & 5.2 & 5.3 & 5.2 & 5.3 & 5.4 & 5.6 & 5.1 & 5.17 & \\
\hline \multirow[t]{2}{*}{6} & 4.4 & 4.6 & 4.3 & 4 & 4 & 4 & 4.4 & 4.3 & 4.4 & 4.5 & 4.29 & \multirow{2}{*}{0.8881} \\
\hline & 4.9 & 5.1 & 4.7 & 4.7 & 4.5 & 4.6 & 5.1 & 5 & 4.8 & 4.9 & 4.83 & \\
\hline \multirow[t]{2}{*}{7} & 5 & 4.9 & 4.5 & 4.6 & 4.8 & 4.8 & 4.7 & 4.4 & 4.7 & 4.3 & 4.67 & \multirow{2}{*}{0.9174} \\
\hline & 5.5 & 5.2 & 5.1 & 5 & 5.2 & 5.1 & 5 & 4.7 & 5.1 & 5 & 5.09 & \\
\hline \multirow[t]{2}{*}{8} & 4.5 & 4.7 & 3.8 & 4.6 & 4.7 & 4.5 & 4.3 & 4.3 & 4.3 & 4.7 & 4.44 & \multirow{2}{*}{0.8827} \\
\hline & 5.1 & 5 & 4.7 & 5 & 5 & 5.3 & 5 & 5 & 5.1 & 5.1 & 5.03 & \\
\hline \multirow[t]{2}{*}{9} & 4.6 & 4.6 & 4.2 & 4.5 & 4.2 & 4.5 & 4.4 & 4.7 & 5 & 4.9 & 4.56 & \multirow{2}{*}{0.8923} \\
\hline & 5.2 & 5.1 & 4.9 & 5 & 4.9 & 4.9 & 4.9 & 5.4 & 5.3 & 5.5 & 5.11 & \\
\hline \multirow[t]{2}{*}{10} & 4.5 & 4.5 & 3.6 & 4.8 & 4.8 & 4.6 & 4.9 & 4.7 & 4.8 & 4.9 & 4.61 & \multirow{2}{*}{0.9092} \\
\hline & 4.7 & 4.8 & 4.4 & 5.1 & 5.4 & 4.9 & 5.5 & 5.3 & 5.2 & 5.4 & 5.07 & \\
\hline \multirow[t]{2}{*}{11} & 4.5 & 4.4 & 4.1 & 4.7 & 4.4 & 4.7 & 4.7 & 4.5 & 4.8 & 4.6 & 4.54 & \multirow{2}{*}{0.9227} \\
\hline & 5 & 4.9 & 4.5 & 5 & 4.9 & 5.1 & 4.9 & 4.9 & 5.1 & 4.9 & 4.92 & \\
\hline \multirow[t]{2}{*}{12} & 4.8 & 5 & 4.9 & 4.6 & 4.7 & 4.7 & 4.1 & 4.7 & 4.8 & 4.9 & 4.72 & \multirow{2}{*}{0.9147} \\
\hline & 5.4 & 5.3 & 5.2 & 5 & 5.3 & 5 & 4.8 & 5.2 & 5.3 & 5.1 & 5.16 & \\
\hline \multirow[t]{2}{*}{13} & 4.6 & 4.6 & 4.7 & 5.1 & 4.5 & 5.1 & 5.2 & 4.9 & 4.9 & 4.6 & 4.82 & \multirow{2}{*}{0.8992} \\
\hline & 5.4 & 5.2 & 5.2 & 5.6 & 5.5 & 5.6 & 5.6 & 5.3 & 5.2 & 5 & 5.36 & \\
\hline 14 & 3.8 & 3.9 & 4.1 & 4 & 4.6 & 4.8 & 4.8 & 4.7 & 4.4 & 4.6 & 4.37 & م ९०04 \\
\hline & 4.3 & 4.4 & 4.7 & 4.5 & 4.4 & 4.9 & 5.2 & 5.3 & 5.7 & 5.2 & 4.86 & 0.0991 \\
\hline 15 & 4.8 & 4.8 & 4.6 & 4.7 & 4.7 & 4.9 & 4.8 & 4.9 & 4.9 & 4.5 & 4.76 & \\
\hline & 5.3 & 5.2 & 5.1 & 5 & 5.1 & 5.4 & 5.2 & 5.4 & 5.4 & 5.2 & 5.23 & 0.9101 \\
\hline 16 & 4.2 & 5.1 & 4.9 & 4.6 & 5 & 4.9 & 4.9 & 5.1 & 4.7 & 5.1 & 4.85 & 00150 \\
\hline & 4.6 & 5.6 & 5.4 & 5 & 5.4 & 5.3 & 5.4 & 5.5 & 5.1 & 5.7 & 5.3 & 0.9150 \\
\hline 17 & 4.7 & 4.7 & 4 & 4.2 & 4.6 & 4.4 & 4.4 & 4.4 & 4.4 & 4.8 & 4.46 & 08884 \\
\hline & 5.1 & 5 & 5 & 4.9 & 5 & 5.3 & 4.6 & 5 & 5.1 & 5.2 & 5.02 & 0.8884 \\
\hline 18 & 5.1 & 4.3 & 4.7 & 4.1 & 4.6 & 4.5 & 4.9 & 4.4 & 5 & 4.9 & 4.65 & \\
\hline & 5.3 & 4.9 & 5.2 & 5 & 5.1 & 5 & 5.5 & 4.9 & 5.4 & 5.3 & 5.16 & 0.9011 \\
\hline 19 & 4.7 & 4.4 & 3.9 & 4.2 & 4.4 & 4.6 & 4.5 & 5 & 4.9 & 4.9 & 4.55 & \\
\hline & 5.2 & 5 & 4.5 & 4.6 & 5 & 5 & 4.9 & 5.3 & 5.2 & 5.4 & 5.01 & 0.9081 \\
\hline 20 & 4.3 & 4.5 & 4.3 & 4.6 & 4.5 & 4.8 & 4.7 & 4.4 & 4.6 & 4.7 & 4.54 & 0 \\
\hline & 4.6 & 5 & 4.5 & 4.9 & 5.1 & 5.2 & 5.1 & 5.1 & 5.4 & 5.2 & 5.01 & 0.9061 \\
\hline 21 & 4.4 & 4.6 & 4.3 & 4.6 & 4.4 & 4.8 & 4.7 & 4.8 & 4.5 & 4.4 & 4.55 & \\
\hline & 4.8 & 5.2 & 4.8 & 5.4 & 4.8 & 5.1 & 5 & 5.1 & 5.1 & 5.1 & 5.04 & 0.9027 \\
\hline 22 & 5 & 4.4 & 4.7 & 4.8 & 4.4 & 5.1 & 4.8 & 5 & 4.8 & 5 & 4.8 & \\
\hline & 5.3 & 4.9 & 5.1 & 5.2 & 4.7 & 5.4 & 5.1 & 5.3 & 5.4 & 5.4 & 5.18 & 0.9266 \\
\hline 23 & 4.8 & 5.1 & 4.8 & 5 & 4.4 & 4.7 & 4.3 & 4.7 & 4.8 & 4.8 & 4.74 & 00111 \\
\hline & 5.3 & 5.4 & 5.2 & 5.4 & 4.8 & 5.1 & 4.9 & 5.3 & 5.2 & 5.4 & 5.2 & 0.9110 \\
\hline 24 & 4.4 & 4.1 & 4.6 & 4.6 & 4.5 & 4.8 & 4.6 & 4.7 & 4.6 & 4.7 & 4.56 & \\
\hline & 4.7 & 4.7 & 4.9 & 5.1 & 5 & 5.1 & 5.1 & 5.2 & 5.3 & 5.2 & 5.03 & 0.9065 \\
\hline 25 & 4.6 & 4.4 & 4.8 & 4.9 & 4.7 & 5 & 4.2 & 4.1 & 4.2 & 4.6 & 4.55 & 08733 \\
\hline & 5.1 & 5.1 & 5.5 & 5.3 & 5.5 & 5.4 & 5 & 5.2 & 4.7 & 5.3 & 5.21 & 0.8733 \\
\hline 26 & 4.8 & 4.6 & 4.7 & 4.1 & 5.1 & 5 & 5.1 & 5.3 & 4.7 & 4.7 & 4.81 & \\
\hline & 5.2 & 4.9 & 5.1 & 4.5 & 5.8 & 5.7 & 5.5 & 5.5 & 5 & 5 & 5.22 & 0.9214 \\
\hline 27 & 5 & 4.7 & 4.8 & 4.8 & 4.9 & 5.2 & 5 & 4.8 & 4.9 & 4.8 & 4.89 & 57 \\
\hline & 5.5 & 5.1 & 5.4 & 5.2 & 5.3 & 5.6 & 5.5 & 5.2 & 5.5 & 5.1 & 5.34 & $0.915 /$ \\
\hline
\end{tabular}

Average RSSI for 5 mm Group- .9049

Table 5: Minimal, Maximal Diameters and RSSI, $5 \mathrm{~mm}$ Stent group. 
Citation: Arena FJ and Arena FA (2013) Intravascular Ultrasound Evaluation of Interwoven Nitinol Stents at Implant. J Vasc Med Surg 1: 116 doi: 10.4172/2329-6925.1000116

Page 5 of 6

\begin{tabular}{|c|c|c|c|c|c|c|c|c|c|c|c|c|}
\hline & 1 & 2 & 3 & 4 & 5 & 6 & 7 & 8 & 9 & 10 & AVE & RSSI \\
\hline Min & 5 & 5 & 4.3 & 4.5 & 4.9 & 5 & 4.6 & 4.9 & 5.5 & 4.7 & 4.84 & \multirow{2}{*}{0.8581} \\
\hline Max & 5.7 & 5.5 & 5 & 4.9 & 5.4 & 5.5 & 6 & 5.9 & 6.6 & 5.9 & 5.64 & \\
\hline \multirow[t]{2}{*}{2} & 5.2 & 5.2 & 6.2 & 4.8 & 5.7 & 5.8 & 5.2 & 3.8 & 4.5 & 5.3 & 5.17 & \multirow{2}{*}{0.8503} \\
\hline & 6.5 & 6.4 & 6.7 & 5.3 & 6.6 & 6.9 & 5.8 & 5.4 & 5.5 & 5.7 & 6.08 & \\
\hline \multirow[t]{2}{*}{3} & 5 & 5.1 & 5.1 & 5.2 & 5 & 5.1 & 4.8 & 4.8 & 5.8 & 5.7 & 5.16 & \multirow{2}{*}{0.9084} \\
\hline & 5.4 & 5.9 & 5.4 & 5.7 & 5.5 & 5.6 & 5.3 & 5.3 & 6.4 & 6.3 & 5.68 & \\
\hline \multirow[t]{2}{*}{4} & 5.4 & 5.2 & 5.3 & 5 & 4.4 & 5 & 4.3 & 4.1 & 4.8 & 5.2 & 4.87 & \multirow{2}{*}{0.8143} \\
\hline & 6 & 5.4 & 5.8 & 6.1 & 5.8 & 5.6 & 6.5 & 6.3 & 6.3 & 6 & 5.98 & \\
\hline \multirow[t]{2}{*}{5} & 5.6 & 5 & 5.5 & 5.6 & 5.5 & 5.4 & 5.2 & 5.6 & 5.6 & 5 & 5.4 & \multirow{2}{*}{0.9} \\
\hline & 6.3 & 5.7 & 6.2 & 6 & 5.8 & 5.9 & 6.1 & 6 & 6.2 & 5.8 & 6 & \\
\hline \multirow[t]{2}{*}{6} & 5.8 & 6.1 & 6 & 5.2 & 5.1 & 5.3 & 5.4 & 5.5 & 5.5 & 5.5 & 5.54 & \multirow{2}{*}{0.9453} \\
\hline & 5.7 & 6 & 5.8 & 6 & 5.6 & 5.7 & 6 & 6 & 5.9 & 5.9 & 5.86 & \\
\hline \multirow[t]{2}{*}{7} & 4.9 & 5.1 & 4.8 & 5.2 & 5 & 5 & 5.9 & 5.8 & 5.4 & 5.5 & 5.26 & \multirow{2}{*}{0.8945} \\
\hline & 5.3 & 5.8 & 5.5 & 5.8 & 5.8 & 5.5 & 6.3 & 6.5 & 6.2 & 6.1 & 5.88 & \\
\hline \multirow[t]{2}{*}{8} & 5.6 & 5.7 & 5.7 & 5.8 & 5.2 & 5.5 & 5.8 & 5.6 & 5.5 & 4.8 & 5.52 & \multirow{2}{*}{0.9004} \\
\hline & 6 & 6.3 & 6.1 & 6.2 & 6 & 6.5 & 6.3 & 6.2 & 5.9 & 5.8 & 6.13 & \\
\hline \multirow[t]{2}{*}{9} & 4.7 & 4.9 & 6 & 5.2 & 4.8 & 5.2 & 5.2 & 5.9 & 6 & 5.7 & 5.36 & \multirow{2}{*}{0.8888} \\
\hline & 5.2 & 5.4 & 6.7 & 6 & 5.4 & 5.7 & 6.1 & 6.8 & 6.6 & 6.4 & 6.03 & \\
\hline \multirow[t]{2}{*}{10} & 4.8 & 5.1 & 5 & 5 & 5 & 5 & 4.5 & 4.9 & 5 & 5 & 4.93 & \multirow{2}{*}{0.9197} \\
\hline & 5.2 & 5.4 & 5.5 & 5.8 & 5.8 & 5.4 & 4.8 & 5.1 & 5.3 & 5.3 & 5.36 & \\
\hline
\end{tabular}

Average RSSI for $6 \mathrm{~mm}$ group- .8880

Table 6: Minimal, Maximal Diameters and RSSI, $6 \mathrm{~mm}$ Stent group.

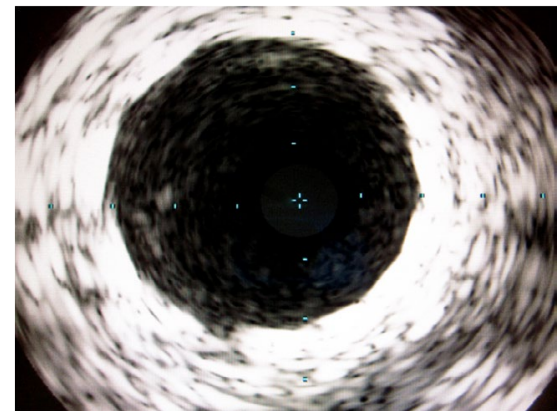

Figure 3a: Interwoven nitinol stent at proximal edge of angioplasty.

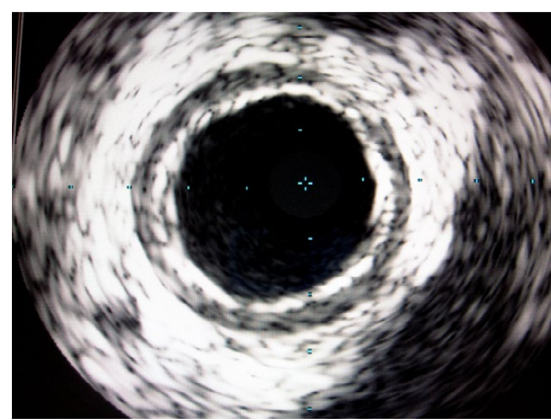

Figure 3b: Native artery just proximal to stent in figure 3, notice the native artery size is smaller than stented area

in the post deployment saving one balloon each case. Anecdotally, the post IVUS sometimes shows vessels are not as large as the stent and are not going to dilate without substantial trauma (Figure $3 a$ and $3 b$ ). The sizing of the vessel is likely to be a major reason why the $6 \mathrm{~mm}$ stents did not perform quite as well as the $5 \mathrm{~mm}$ stents in this study. The additional information provided by the RSSI calculation supports the observation that these stents deploy with consistent round lumens. We saw only one instance where the measured lumen was less than $50 \%$ of the projected nominal area, (Figure 4) and no stent deployed in a crescent moon shape as has been seen with traditional nitinol designs. 
Citation: Arena FJ and Arena FA (2013) Intravascular Ultrasound Evaluation of Interwoven Nitinol Stents at Implant. J Vasc Med Surg 1: 116 doi: 10.4172/2329-6925.1000116

Page 6 of 6

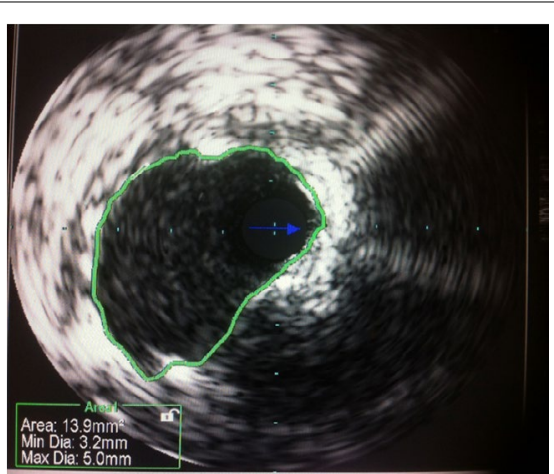

Figure 4: A short segment of $6 \mathrm{~mm}$ interwoven nitinol stent in a heavily calcified artery (over 200 degree arc of calcium is noted) achieving only $49.1 \%$ of its nominal lumen.

\section{Conclusions}

In this study, interwoven Nitinol stents perform well at implant with consistent SER across multiple stent sizes when used in the SFA and Popliteal arteries. The RSSI data showed they deployed with relatively round shapes and generally did not appear to suffer substantially from external compression.

\section{References}

1. Schillinger M, Sabeti S, Loewe C, Dick P, Amighi J, et al. (2006) Balloon angioplasty versus implantation of nitinol stents in the superficial femoral artery. N Engl J Med 354: 1879-1888.

2. Albiero R, Rau T, Schluter M, Di Mario C, Reimers B, et al. (1997) Comparison of immediate and intermediate-term results of intravascular ultrasound versus angiography-guided Palmaz-Schatz stent implantation in matched lesions. Circulation 96: 2997-3005.

3. Kojiro M, Kenichi, F, Daizo K (2012) The Impact of Postprocedural Intravascular Ultrasound Findings on Long-Term Results Following Self-Expanding Nitinol Stenting in the Superficial Femoral Artery. ACC Moderated Poster contributions. Category 37, Presentation number: 1122-1133.

4. de Jaegere P, Mudra H, Figulla H, Almagor Y, Doucet S, et al. (1998) Intravascular ultrasound-guided optimized stent deployment. Immediate and 6 months clinical and angiographic results from the Multicenter Ultrasound Stenting in Coronaries Study (MUSIC Study). Eur Heart J 19: 1214-1223.

5. de Feyter PJ, Kay P, Disco C, Serruys PW (1999) Reference chart derived from post-stent-implantation intravascular ultrasound predictors of 6-month expected restenosis on quantitative coronary angiography. Circulation 100: 1777-1783.

6. Oemrawsingh PV, Mintz GS, Schalij MJ, Zwinderman AH, Jukema JW, et al. (2003) Intravascular ultrasound guidance improves angiographic and clinical outcome of stent implantation for long coronary artery stenoses: final results of a randomized comparison with angiographic guidance (TULIP Study). Circulation 107: 62-67.

7. White RA, Donayre CE, Kopchok GE, Walot I, Mehringer CM (1997) Utility of intravascular ultrasound in peripheral interventions. Tex Heart Inst J 24: 28-34.

8. Miki K, Fujii K, Kawasaki D (2012) The impact of Post-procedural intravascular ultrasound findings on the long term results following self-expanding nitinol stenting in the Superficial Femoral Artery. J Am Coll Cardiol; 59(13s1): E2112. S0735-1097(12)62113-5.

9. Scheinert D, Grummt L, Piorkowski M, Sax J, Scheinert S, et al. (2011) A novel self-expanding interwovennitinol stent for complex femoropopliteal lesions: 24-month results of the SUPERA SFA registry. J Endovasc Ther 18: 745-752.

10. Scheinert D, Werner M, Scheinert S, Paetzold A, Banning-Eichenseer U, et al. (2013) Treatment of Complex Atherosclerotic Popliteal Artery Disease With a New Self-Expanding Interwoven Nitinol Stent: 12-Month Results of the Leipzig SUPERA Popliteal Artery Stent Registry. JACC Cardiovasc Interv 6: 65-71. 